\title{
Comparative study of subculture, Gram staining and acridine orange staining for early detection of positive blood cultures
}

\author{
GEORGES MASCART, FRANCOIS BERTRAND, PHILIPPE MASCART \\ From the Department of Microbiology, Edith Cavell Clinic, 32 rue Edith Cavell, 1180 Brussels, Belgium
}

SUMMARY In view of the importance of a rapid aetiological diagnosis in septicaemia, we compared the results of subculture, Gram staining and acridine orange staining in the detection of positive blood cultures. The study was based on 1013 blood cultures of which 138 were positive by culture. The three techniques were applied $12 \mathrm{~h}$ after the specimen was taken in 210 instances, at $24 \mathrm{~h}$ in 540 instances and after $48 \mathrm{~h}$ in 525 . We were able to demonstrate the value of direct examination. Staining with acridine orange yields more positive results than Gram staining and is also simpler.

Early recognition of positive blood cultures is very important for microbiology laboratories. Some laboratories have solved the problem by using the BACTEC system which is based on the utilisation of ${ }^{14} \mathrm{C}$-containing substrates by multiplying bacteria. Measurement of the ${ }^{14} \mathrm{C}$-containing $\mathrm{CO}_{2}$ produced allows early detection of positive blood cultures. In our laboratory we have relied on more conventional methods but wanted to try out the acridine orange technique recently described by Kronwall and Myre ${ }^{1}$ and applied by McCarthy and Senne to blood cultures. $^{2}$

\section{Material and methods}

Our study was based on 1013 blood cultures from the Edith Cavell Clinic and the Joseph Bracops Hospital Center and was limited to the aerobic bottles. After disinfecting the skin with iodine-alcohol or isobetadine, $20 \mathrm{ml}$ of blood were taken and after changing the needle, half was injected in the aerobic bottle (BCP Roche-157, avenue du Roi, 1060 Brussels) which contained $70 \mathrm{ml}$ of Brain Heart Infusion medium with Liquoid as the anticoagulant and the other half into the anaerobic bottle (Schaedler Bio Merieux-91, avenue du Diamant, 1040 Brussels).

The three following methods were compared:

1 Subculture After taking the specimen, there was between 4 and $24 \mathrm{~h}$ before we received aerobic

Accepted for publication 29 December 1982 bottles at our laboratory and so these were incubated in peripheral laboratories. On receipt of the bottle by the microbiological laboratory, the cap was removed and a BCP "Slide" was substituted (BCP Roche-157 avenue du Roi-1060 Brusssels). This is a small plastic cylinder enclosing a plate with three different agar media on it (Mac Conkey, Chocolate and Sabouraud). Inverting the bottle briefly fills the device and seeds the media. The media are examined after $24 \mathrm{~h}$ and if negative the plate is seeded again. Incubation is at $35^{\circ} \mathrm{C}$ in normal atmosphere.

2 Gram staining After mixing the culture in the bottle, the BCP Slide is unscrewed and $0.5 \mathrm{ml}$ removed with a sterile syringe and needle; a drop is placed on a microscope slide for Gram staining by the classical technique.

3 Acridine orange staining (McCarthy's method) Preparation of the acridine orange solution (AO): dissolve $100 \mathrm{mg}$ acridine orange (Fischer Scientific) in $100 \mathrm{ml}$ distilled water. This stock solution should be stored in the dark at $4^{\circ} \mathrm{C}$ and under these conditions will last six months. A working solution needs to be prepared daily by making a $1 / 100$ dilution of the stock in Walpole's acetate buffer $(0.2$

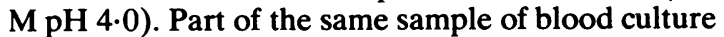
withdrawn for Gram staining is also used for staining with acridine orange. Fluorescence slides with eight wells are used, one small drop of the sample from each specimen being placed in each well and the excess reaspirated with the syringe so that only a thin film remains. A positive control is included on 
each slide and the slide is left to dry at $37^{\circ} \mathrm{C}$ for 30 min. The slides are then fixed in absolute methyl alcohol for $2 \mathrm{~min} ; 25 \mu \mathrm{l}$ of the working solution of AO are put in each well for $1 \mathrm{~min}$. The slide is then rinsed with water and dried. Under these conditions bacteria are stained light orange, white cells are green and red cells are unstained.

Each of the three techniques was applied as far as possible at 12, 24 and $48 \mathrm{~h}$ after the specimen was taken. The technician reading the AO slides did not know the results of the Gram stains or the subcultures.

\section{Results}

Our study is based on 1013 blood cultures of which 138 were positive by culture. The bacteria identified in the positive blood cultures are reported in Table 1.

Gram staining, acridine staining and subculture were run simultaneously on:

-210 samples after $12 \mathrm{~h}$ inoculation,

-540 samples after $24 \mathrm{~h}$ inoculation,

-525 samples after $48 \mathrm{~h}$ inoculation.

Direct examination was positive: (Table 2)

-in 4 cases by Gram staining only,

-in 25 cases by acridine staining only,

-in 74 cases simultaneously by both methods.

Table 1 Organisms isolated in 1013 consecutive blood cultures

\begin{tabular}{llr}
\hline Gram-negative bacilli & Escherichia coli & 30 \\
& Klebsiella & 5 \\
& Salmonella & 3 \\
& Proteus mirabilis & 1 \\
& Proteus morganii & 1 \\
& Pseudomonas aeruginosa & 1 \\
& Enterobacter agglomerans & 1 \\
Gram-positive bacilli & Clostridium perfringens & 1 \\
& Corynebacterium & 1 \\
Gram-positive cocci & Fusobacterium & 1 \\
& Staphylococcus epidermidis & 51 \\
& Staphylococcus aureus & 32 \\
& Pneumococcus & 5 \\
& Microccus & 3 \\
& Streptococcus & 1 \\
& Enterococcus & 1 \\
\hline
\end{tabular}

Table 2 Results of Gram stain, acridine orange stain and subcultures of 1013 consecutive blood cultures

\begin{tabular}{lr}
\hline Total number of positive cultures & 138 \\
Results given first by direct examination* & 103 \\
- By acridine only & 25 \\
-By Gram only & 4 \\
-By Gram and acridine & 74 \\
Results not given by direct examination & 35 \\
-False positive by Gram & 3 \\
-False positive by acridine & 3 \\
\hline "10 cultures became positive more than 24 hours after direct examination \\
revealed a positive result.
\end{tabular}

Table 3 Results of direct examination (false-positive excluded)

\begin{tabular}{llcc}
\hline Culture-positive & $12 h$ & $24 h$ & $48 h$ \\
\hline Acridine only & 5 & 16 & 4 \\
Gram only & 3 & 1 & 0 \\
Acridine and Gram & 4 & 37 & 33 \\
\hline
\end{tabular}

Cultures became positive more than $24 \mathrm{~h}$ after direct examination in 10 cases. In 35 cases, the result was not given by direct examination.

Moreover, we must mention three false-positive results by Gram and three other by acridine staining.

Altogether 108 (6 false-positive) of 1013 blood cultures were reported as positive at the time subcultures were made, resulting in earlier reporting.

Direct examination was positive at $12 \mathrm{~h}$ in 12 cases of which five were positive with acridine and negative with Gram staining. At 24 h, 56 cases were positive (two false-positive) of which 16 with acridine only and at $48 \mathrm{~h}, 40$ were positive (three false-positive) of which four with acridine only (Table 3).

The increased sensitivity of the acridine staining method as compared to the Gram staining is more striking at 12 and $24 \mathrm{~h}$ and this improves an earlier diagnosis.

In 10 cases the delay between the positive direct examination and the positive culture was more than $24 \mathrm{~h}$.

\section{Discussion}

The classical way of detecting that blood cultures are positive is by looking for turbidity or haemolysis and by subculture. This leads to significant delays in reporting which many authors have tried to reduce by using new techniques such as radiometric methods ${ }^{3-5}$ or centrifugation methods. ${ }^{67}$ The benefit of using new approaches is substantial when one bears in mind that certain organisms (meningococcus, gonococcus, $H$ influenzae, Bacteroides, Fusobacterium, Pseudomonas spp and Strep pneumoniae) can multiply among the sedimented blood cells without producing any visible turbidity. ${ }^{89}$ To surmount this difficulty some authors have recommended making subcultures routinely at $48 \mathrm{~h}$ and at 5 and 7 days. $^{89}$ Blazevic ${ }^{8}$ on the other hand, bearing in mind that subculture will delay the reporting of any result for another day, recommends doing a Gram stain routinely, at any rate after the first day incubation. As a result, $23 \%$ of her positive blood cultures were detected first in the Gram stain. These figures were not however confirmed by Hall. ${ }^{9}$

Mirett, Reller and Lauer reported equally encouraging results at the Congress of the American 
Society for Microbiology in Miami in May 1980. They found that the sensitivity of Gram and acridine orange staining were respectively of $34 \%$ and $49 \%$. The specificity was respectively of $99.4 \%$ and $98.9 \%$. The authors concluded that the orange technique was a simple and sensitive screening procedure for blood cultures.

Recently McCarthy and Senne ${ }^{2}$ examined with the acridine orange technique 2704 blood cultures which showed no sign of turbidity after $24 \mathrm{~h}$. Of 103 positive bottles initially detected either by $\mathrm{AO}$ or subculture or by both; $85(78 \%)$ were by both methods at the same time, $14(12.8 \%)$ by subculture alone and $10(9.2 \%)$ by AO alone.

We used for our subcultures the "Roche" system which is not classical but is very easy and saves time. Recent reports consider this method as being equivalent to the classical one. ${ }^{10}$ Our results showed immediately that direct examination with Gram and AO stains allowed us to recognise positive blood cultures considerably earlier than we would otherwise have done. In 103 cases (74.6\%) we were able to report that a culture was positive at least one day earlier. In 74 cases $(53.6 \%)$ the Gram and AO were both positive; in $25(18.1 \%)$ the $\mathrm{AO}$ was positive but the Gram negative; and in only $4(2.8 \%)$ cases were the Gram positive and the AO negative.

Staining with acridine orange is clearly superior to the classical Gram stain. As in addition it only gave three false-positives the specificity of the AO method can be regarded as excellent and equivalent to that of Gram staining.

Our technician required one hour per day for direct examination. We think that the cost benefit ratio is in favour of this technique. During our study we had no more contaminants than usual. The high percentage of Staphylococcus epidermidis and Micrococcus is related to the fact that blood cultures were taken by young medical students whose technique was suspect.
In conclusion, it seems to us that it is essential to carry out direct microscopical examinations of all blood cultures in the first two days after they are taken (the period during which most positive cultures can be detected) and if only one staining method is practical, we recommend acridine orange.

\section{References}

' Kronvall G, Myre E. Differential staining of bacteria in clinical specimens using acridine orange buffered at low pH. Acta Pathol Microbiol Scand $[B]$ 1977;85:249-54.

${ }^{2}$ McCarthy LR, Senne JE. Evaluation of acridine orange stain for detection of microorganisms in blood cultures. J Clin Microbiol 1980;11:281-5.

${ }^{3}$ Deland F, Wagner Jr HN. Automated radiometric detection of bacterial growth in blood cultures. J Lab Clin Med 1970;65:529-34.

4 Renner ED, Catheridge LA, Washington II JA. Evaluation of radiometric system for detecting bacteremia. Appl Microbiol 1973;26:368-72.

${ }^{5}$ Brooks K, Sodeman T. Rapid detection of bacteremia by a radiometric system. Am J Clin Pathol 1974;61:859-66.

' Dorn GL, Smith K. New centrifugation blood culture device. $J$ Clin Microbiol 1978;7:52-4.

' Hilbery Sh M. Use of centrifugation method of blood culturing in a community acute care hospital. Lab Med 1979;10:674-8.

- Blazevic DJ, Stemper JE, Matsen JM. Comparison of macroscopic examination, routine Gram stains and routine subcultures in the initial detection of positive blood cultures. Appl Microbiol 1974;27:537-9.

' Hall M, Warren E, Washington II JA. Comparison of two liquid blood culture media containing sodium polyane etholsulfonate: tryptic soy and Columbia. Appl Microbiol 1974;27:699-702.

${ }^{10}$ Bryan LE. Comparison of a slide blood culture system with a supplemented peptone broth culture method. J Clin Microbiol 1981;14:389-92.

Requests for reprints to: Dr G Mascart, Rue du Châtelain, 32-Brussels 1050-Belgium. 